\title{
SQUARE COMPLEXES AND SIMPLICIAL NONPOSITIVE CURVATURE
}

\author{
TOMASZ ELSNER AND PIOTR PRZYTYCKI \\ (Communicated by Alexander N. Dranishnikov)
}

\begin{abstract}
We prove that each nonpositively curved square $\mathcal{V H}$-complex can be turned functorially into a locally 6-large simplicial complex of the same homotopy type. It follows that any group acting properly and cocompactly on a $\mathrm{CAT}(0)$ square $\mathcal{V} \mathcal{H}$-complex is systolic. In particular, the product of two finitely generated free groups is systolic, which answers a question of Daniel Wise. On the other hand, we exhibit an example of a non- $\mathcal{V H}$ nonpositively curved square complex whose fundamental group is neither systolic nor even virtually systolic.
\end{abstract}

\section{INTRODUCTION}

In this note we compare nonpositively curved square $\mathcal{V H}$-complexes (introduced in [Wis96]) and locally 6-large simplical complexes (introduced in [JŚ06]).

The paper is divided into two parts. In Section 3 we provide a functorial construction turning a nonpositively curved square $\mathcal{V H}$-complex into a locally 6-large simplicial complex of the same homotopy type (in particular, turning a CAT $(0)$ $\mathcal{V H}$-complex into a systolic complex). The main application of the construction is:

Theorem 1.1 (see Corollary [3.6). The fundamental group of a compact nonpositively curved $\mathcal{V H}$-complex is systolic.

The first application of Theorem 1.1 is the answer to a question posed by Daniel Wise in Wis05:

Corollary 1.2. The product of two finitely generated free groups is systolic.

We also obtain a series of consequences of Theorem 1.1 by applying it to the examples of nonpositively curved $\mathcal{V H}$-complexes (some with exotic properties) given by Daniel Wise in Wis96 and Wis07.

Corollary 1.3 (cf. [Wis96, Corollary 2.8]). The fundamental group of an alternating knot complement is systolic.

Corollary 1.4 (cf. Wis07, Theorem 7.5]). There exists a systolic group which is not residually finite.

One can arrange for an even stronger property:

Received by the editors July 20, 2011 and, in revised form, November 21, 2011.

2010 Mathematics Subject Classification. Primary 20F65.

The first author was partially supported by MNiSW grant N N201 541738.

The second author was partially supported by MNiSW grant N N201 541738 and the Foundation for Polish Science. 
Corollary 1.5 (cf. Wis07, Theorem 8.10]). There exists an infinite systolic group which has no finite-index subgroups.

Corollary 1.6 (cf. [BM00, Section 6]). There exists an infinite simple systolic group.

In Section 4 we show that the $\mathcal{V H}$-hypothesis in Theorem 1.1 is necessary:

Theorem 1.7 (see Theorem 4.2). There exists a compact nonpositively curved square complex whose fundamental group is not systolic or even virtually systolic.

\section{Square COMPleXes AND SIMPlex COMPleXeS}

First we describe locally 6-large and systolic complexes. For the sake of generality we use the notion of a simplex complex in place of a simplicial complex. A simplex complex is essentially a complex built from simplices by gluing them together along subsimplices. The notion arises quite naturally, as the quotient $X / G$ of a simplicial complex $X$ by a group $G$ acting freely on $X$ is not always a simplicial complex but of course is a simplex complex.

Definition 2.1. A simplex complex is a set $\mathcal{S}$ of affine simplices together with a set $\mathcal{E}$ (closed under compositions) of affine embeddings of simplices of $\mathcal{S}$ onto the faces of simplices of $\mathcal{S}$ (attaching maps) such that for any proper face $\tau$ of any simplex $\sigma \in \mathcal{S}$ there is precisely one attaching map onto $\tau$.

The link of a vertex $v$ in a simplex complex $X=(\mathcal{S}, \mathcal{E})$ is the simplex complex $X_{v}=\left(\mathcal{S}_{v}, \mathcal{E}_{v}\right)$ where the set $\mathcal{S}_{v}$ is obtained by taking for each attaching map $\phi_{v, \tau}: v \rightarrow \tau$ the facet of $\tau$ opposite to the image of $v$ and $\mathcal{E}_{v}$ is the set of restrictions of the maps in $\mathcal{E}$.

Definition 2.2. A simplicial complex $X$ is flag if the vertices of any complete subgraph (a clique) in $X^{(1)}$ span a simplex of $X$.

A cycle without diagonals in a simplicial complex $X$ is an embedded closed combinatorial path $\gamma$ such that no edge in $X$ connects nonconsecutive vertices of $\gamma$.

A simplex complex is locally 6-large if all of its vertex links are flag (in particular, simplicial) and do not contain cycles of length 4 or 5 without diagonals.

A simply connected locally 6-large simplex complex is called systolic (i.e. systolic complexes are the universal coverings of connected locally 6-large complexes).

A group admitting a proper and cocompact action on a systolic complex is systolic.

The original definition of local 6-largeness in JŚ07 requires that we check the flagness and the absence of short cycles without diagonals for the link of every simplex. However, for higher-dimensional simplices it is a direct consequence of those properties for the links of the vertices.

Notice that a locally 6-large simplex complex is a simplicial complex unless it contains a loop (an edge joining a vertex to itself) or a double edge. Thus the above definition of a locally 6-large complex is slightly more general than the one in [JŚ07]; nevertheless the definition of a systolic complex fully coincides with the one in JŚ́07.

Having defined nonpositively curved simplex complexes, we now define nonpositively curved square complexes. Similarly as for simplex complexes, we allow cells in square complexes not to be embedded. The formal definition of a square complex 
is the same as of a simplex complex except for putting vertices, edges and squares in place of simplices. The only thing that needs to be rephrased is the definition of the link.

Definition 2.3. The link of a vertex $v$ in a square complex $X=(\mathcal{S}, \mathcal{E})$ is a 1dimensional simplex complex $X_{v}=\left(\mathcal{S}_{v}, \mathcal{E}_{v}\right)$ (a graph), where $\mathcal{S}_{v}$ is obtained by taking for each attaching map $\phi_{v, \sigma}: v \rightarrow \sigma$ the vertex of $\sigma$ opposite to $v$ (if $\sigma$ is an edge) or the diagonal of $\sigma$ opposite to $v$ (if $\sigma$ is a square) and $\mathcal{E}_{v}$ is the set of restrictions of the maps in $\mathcal{E}$.

A square complex is called a $\mathcal{V H}$-complex if its 1-cells can be partitioned into two classes $V$ and $H$ called vertical and horizontal edges, respectively, and the attaching map of each square alternates between the edges of $V$ and $H$. In other words, the link of each vertex is a bipartite graph with independent sets of vertices coming from edges of $V$ and $H$.

Note that the link of a vertex in a $\mathcal{V H}$-complex may have double edges.

Definition 2.4. A square complex is nonpositively curved (or locally CAT(0)) if the link of any vertex does not contain an embedded combinatorial cycle of length less than 4 . For a $\mathcal{V H}$-complex this reduces to the property that there are no double edges in the links of vertices.

Example 2.5. The product of two trees is a CAT $(0) \mathcal{V H}$-complex. If a group acts freely by isometries on the product of two trees and preserves the coordinates, then the quotient square complex is a nonpositively curved $\mathcal{V H}$-complex.

\section{Nonpositively CURVEd $\mathcal{V H}$-COMplexes are Systolic}

Our main construction yields a way of turning a nonpositively curved $\mathcal{V H}$ complex into a locally 6-large simplex complex.

Construction 3.1. Let $X$ be a $\mathcal{V H}$-complex with the sets $E_{\mathcal{V}}$ and $E_{\mathcal{H}}$ of vertical and horizontal edges, respectively. Denote by $V$ and $S$ the sets of vertices and squares of $X$, respectively. We construct an associated simplex complex $X^{*}$, called the simplexification of $X$, which has the same homotopy type as $X$.

First we divide each vertical edge $e \in E_{\mathcal{V}}$ in two and subdivide each square $s \in S$ into six triangles, as in Figure 1(a), obtaining a simplex complex $\hat{X}$ (a triangulation of $X$ ). The vertices of $\hat{X}$ (which will correspond to the vertices of $\left.X^{*}\right)$ are in bijective correspondence with the elements of $V \cup E_{\mathcal{V}} \cup S$. We denote those vertices by $v^{*}, e^{*}, s^{*}$, for $v \in V, e \in E_{\mathcal{V}}, s \in S$, respectively.

(a)

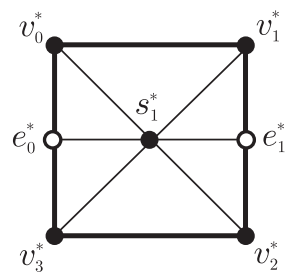

(b)

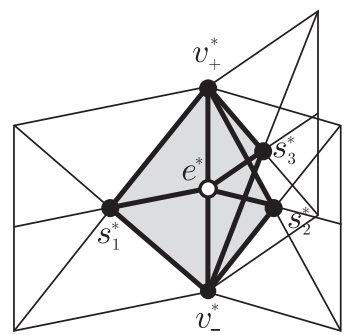

(c)

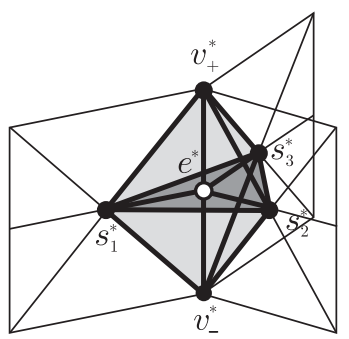

Figure 1. (a) the triangulation of $X$

(b) $\hat{Y}_{e} \subset \hat{X}$

(c) $Y_{e}^{*} \subset X^{*}$ 
The link of $e^{*}$ in $\hat{X}$ is isomorphic to the suspension of a set of $n=\operatorname{deg}(e)$ points, where $\operatorname{deg}(e)$ is the number of squares $s \in S$ with a vertical edge $e$ (counted with multiplicities; i.e. a square with both vertical edges equal to $e$ is counted twice). The union $\hat{Y}_{e}$ of all the simplices of $\hat{X}$ containing the vertex $e^{*}$ is isomorphic to the suspension of an $n$-pod, where some pairs of vertices may be identified.

The complex $X^{*}$ is obtained from $\hat{X}$ by attaching $(n+1)$-simplices $\sigma_{v}^{+}=$ $\left(v_{+}^{*}, e^{*}, s_{1}^{*}, \ldots, s_{n}^{*}\right)$ and $\sigma_{v}^{-}=\left(v_{-}^{*}, e^{*}, s_{1}^{*}, \ldots, s_{n}^{*}\right)$ for each vertex $e^{*}$, where $v_{+}$and $v_{-}$are the endpoints of the edge $e \in E_{\mathcal{V}}$ and $s_{1}, \ldots, s_{n}$ are the squares adjacent to the vertical edge $e \in E_{\mathcal{V}}$ (counted with multiplicities).

The link of $e^{*}$ in $X^{*}$ is the suspension of an $(n-1)$-simplex, and the union $Y_{e}^{*}$ of all the simplices of $X^{*}$ containing the vertex $e^{*}$ is isomorphic to the suspension of an $n$-simplex, where some pairs of vertices may be identified: $v_{+}^{*}$ and $v_{-}^{*}$ are identified if the edge $e \in E_{\mathcal{V}}$ is a loop, and $s_{i}$ and $s_{j}$ are identified if there is a square $s \in S$ with both vertical edges equal to $e$. Notice that $Y_{e_{p}}^{*}$ and $Y_{e_{q}}^{*}$ for different $e_{p}, e_{q} \in E_{\mathcal{V}}$ are disjoint except possibly at $v_{ \pm}^{*}$ and $s_{i}^{*}$.

Proposition 3.2. A $\mathcal{V H}$-complex $X$ and its simplexification $X^{*}$ have the same homotopy type.

Proof. As the triangulation $\hat{X}$ of $X$ embeds into $X^{*}$, we only need to prove that $X^{*}$ deformation retracts onto $\hat{X}$. The deformation retraction arises from collapsing along free faces, and this is possible because of the essential disjointness of the various $Y_{e_{i}}^{*}$ in $X$ as elaborated above. Precisely since for distinct $e_{0}, e_{1} \in E_{\mathcal{V}}$ we have $Y_{e_{0}}^{*} \cap Y_{e_{1}}^{*} \subset \hat{X}$, it is enough to show that for any $e \in E_{\mathcal{V}}$ the complex $Y_{e}^{*}$ deformation retracts onto $Y_{e}^{*} \cap \hat{X}=\hat{Y}_{e}$.

If $Y_{e}^{*}$ is a simplicial complex (i.e. the suspension of the simplex $\sigma\left(s_{1}^{*}, \ldots, s_{n}^{*}, e^{*}\right)$ ), then denoting by $S$ the suspension and by $C$ the cone operator, we have

$$
\hat{Y}_{e}=S\left(C\left(\left\{s_{1}^{*}, \ldots, s_{n}^{*}\right\}\right)\right) \subset S\left(C\left(\sigma\left(s_{1}^{*}, \ldots, s_{n}^{*}\right)\right)\right)=Y_{e}^{*},
$$

where $\sigma\left(s_{1}^{*}, \ldots, s_{n}^{*}\right)$ is the simplex with vertices $s_{1}^{*}, \ldots, s_{n}^{*}$.

Consider the retraction $r: C\left(\sigma\left(s_{1}^{*}, \ldots, s_{n}^{*}\right)\right) \rightarrow C\left(\left\{s_{1}^{*}, \ldots, s_{n}^{*}\right\}\right)$ defined to be the affine extension of the map from the first barycentric subdivision, which preserves the subcomplex $C\left(\left\{s_{1}^{*}, \ldots, s_{n}^{*}\right\}\right)$ and maps the barycentres of the remaining simplices to the cone vertex. It is easy to see that $r$ can be extended to a deformation retraction. By suspending the deformation retraction, we obtain a deformation retraction from $Y_{e}^{*}$ onto $\hat{Y}_{e}$.

If $Y_{e}^{*}$ is a simplex complex which is not simplicial, then it is a quotient space of the suspension of a simplex obtained by identifying some pairs of vertices. In that case the deformation retraction from $Y_{e}^{*}$ onto $\hat{Y}_{e}$ is the quotient of the map described above.

Remark 3.3. Note that Construction 3.1 is functorial. Namely, let $f: X \rightarrow Y$ be a combinatorial map between $\mathcal{V H}$-complexes (i.e. mapping cells onto cells of the same dimension, in our case mapping edges to edges and squares to squares). Assume also that $f$ preserves the sets of vertical and horizontal edges. Then $f$ induces a canonical combinatorial map $f^{*}: X^{*} \rightarrow Y^{*}$. Moreover, we have $(f \circ g)^{*}=f^{*} \circ g^{*}$ and $i d^{*}=i d$.

In particular, if $f$ is a combinatorial isomorphism, then so is $f^{*}$. Finally, note that if a group $G$ acts properly and/or cocompactly on $X$, then its induced action on $X^{*}$ is proper and/or cocompact. 
We are now ready for our main result.

Theorem 3.4. If $X$ is a nonpositively curved $\mathcal{V H}$-complex, then its simplexification $X^{*}$ is locally 6-large.

Before giving the proof, we list a few consequences, obtained by applying Proposition 3.2 and Remark 3.3 .

Corollary 3.5. If $X$ is a $\mathrm{CAT}(0) \mathcal{V H}$-complex, then its simplexification $X^{*}$ is systolic. If $G$ acts properly and cocompactly on $X$, then $G$ is systolic.

There are two notable applications of Corollary 3.5 .

Corollary 3.6 (Theorem 1.1). The fundamental group of a compact nonpositively curved $\mathcal{V H}$-complex is systolic.

The second application promotes Wise's aperiodic flat construction (Wis03, Theorem 3.2]) into the systolic setting.

Definition 3.7. A flat in a systolic complex $X$ is a subcomplex $\mathbb{E}_{\triangle}^{2} \subset X$ which is isomorphic to the equilaterally triangulated plane (the triangulation with 6 triangles adjacent to each vertex) and whose 1-skeleton is isometrically embedded into $X^{(1)}$ (with the combinatorial metric).

Corollary 3.8. There exists a compact locally 6-large simplex complex whose (systolic) universal cover contains a flat that is not the limit of a sequence of periodic flats.

It remains to prove our main result.

Proof of Theorem 3.4. We need to check that the link of any vertex in $X^{*}$ is flag and does not contain cycles of length 4 or 5 without diagonals.

For $e \in E_{\mathcal{V}}$, the link of $e^{*}$ is the suspension of a simplex, so it is flag and contains no cycle without diagonals.

For $s \in S$, the link of $s^{*}$ is isomorphic to the union of two suspensions of simplices, $S\left(\sigma_{n_{0}-1}\right)$ and $S\left(\sigma_{n_{1}-1}\right)$, together with edges joining their top and bottom vertices. Here $n_{i}=\operatorname{deg}\left(e_{i}\right)$, where $e_{0}$ and $e_{1}$ are the vertical edges of $s$ (the case $n_{0}=3$, $n_{1}=2$ is depicted in Figure 2(b)). It is clear that it is flag and any cycle without diagonals in that link has length at least 6 .

(a)

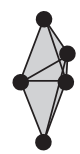

(b)

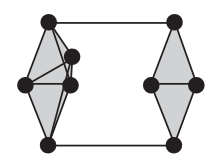

(c)

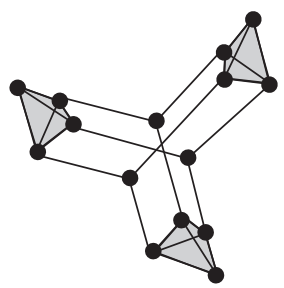

(b) $s^{*}$ (c) $v^{*}$

For $v \in V$, the link of $v^{*}$ is the union of $n_{e}$-simplices for all vertical edges $e \in E_{\mathcal{V}}$ issuing from $v$ and $n_{e}$-pods for all horizontal edges $e \in E_{\mathcal{H}}$ issuing from $v$ $\left(n_{e}=\operatorname{deg}(e)\right)$, with each endpoint of each $n_{e}$-pod identified with a different vertex of one of the simplices. It is clear that such a link is flag and any cycle without 
diagonals in that link passes through at least two $n_{e}$-pods and two simplices, which makes its length at least 6 (Figure 2(c) depicts the link of $v^{*}$ in the case when the neighbourhood of $v \in V$ in $X$ is the product of two tripods).

\section{EXAmples of NONPOSITIVELY CURVED SQUARE COMPLEXES WHICH ARE NOT SYSTOLIC}

In the next part of the paper we show that Theorem 3.4 cannot be improved to include all nonpositively curved square complexes. Namely, we construct an example of a compact nonpositively curved square complex whose fundamental group is not systolic. Later, we use that example to provide a compact nonpositively curved square complex whose fundamental group is neither systolic nor even virtually systolic (Theorem 1.7).

Let $K$ be the square complex presented in Figure 3, built from two squares. It has only one vertex, and the link at this vertex is shown in Figure 4. Thus we see that $K$ is a nonpositively curved square complex but not a $\mathcal{V H}$-complex. We will show that $\pi_{1}(K)$ is not a systolic group.
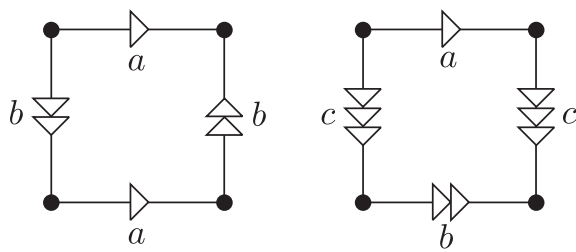

FiguRE 3 . The nonpositively curved square complex $K$

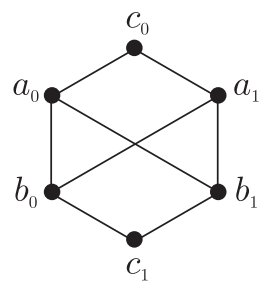

Figure 4 . The link of $K$ at the only vertex

Theorem 4.1. The group $\pi_{1}(K)$ is not systolic.

Proof. The group

$$
\pi_{1}(K)=\left\langle a, b, c \mid b a=a b^{-1}, a=c b c^{-1}\right\rangle
$$

is an HNN-extension of the fundamental group of a Klein bottle, so it has a subgroup $H=\langle a, b\rangle$ which is isomorphic to the fundamental group of a Klein bottle, and in particular $H$ is virtually $\mathbb{Z}^{2}$.

Suppose $\pi_{1}(K)$ is systolic, i.e. acts properly and cocompactly on some systolic simplicial complex $X$. As a corollary from the systolic flat torus theorem (precisely by Corollary 6.2(1) together with Theorem 5.4 in [Els09]) we have that $H$, as a 
torsion free virtually $\mathbb{Z}^{2}$ group, acts properly on a systolic flat in $X$ (see Definition 3.7). If the fundamental group of a Klein bottle $\left\langle a, b \mid b a=a b^{-1}\right\rangle$ acts properly by isometries on the Euclidean plane, then $a$ acts as a glide reflection and $b$ as a translation in the direction perpendicular to the glide reflection axis. In our case the plane has the additional combinatorial structure of $\mathbb{E}_{\triangle}^{2}$. There are only two (up to an isomorphism of $\mathbb{E}_{\triangle}^{2}$ ) possible axes for a glide reflection acting on $\mathbb{E}_{\triangle}^{2}: k$ and $l$. If the axis of the glide reflection $a$ is $l$, then the direction of the translation $b$ is $k$, and vice versa.

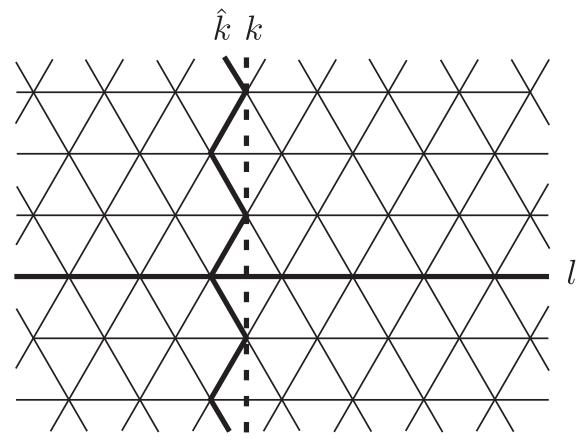

FiguRE 5. $\mathbb{E}_{\triangle}^{2}$

The elements $a^{2}$ and $b^{2}$ act by translations on $\mathbb{E}_{\triangle}^{2}$ (with axes $k$ and $l$ ). The 1-skeleton of $\mathbb{E}_{\triangle}^{2}$ with the combinatorial metric is isometrically embedded into the 1-skeleton of $X$ (by Definition 3.7), so the lines $\hat{k}$ and $l$ (indicated in Figure 5) are invariant combinatorial geodesics for $a^{2}$ and $b^{2}$. By [Els10, Proposition 3.11] the geodesic $l$ is quasi-convex in the 1 -skeleton of $X$ equipped with the combinatorial metric (i.e. any geodesic in $X^{(1)}$ with both endpoints on $l$ is contained in the $\delta$ neighbourhood of $l$, for some universal $\delta$ ). However, the geodesic $\hat{k}$ is clearly not quasi-convex, as every vertex of $\mathbb{E}_{\triangle}^{2}$ lies on some geodesic with both endpoints on $\hat{k}$.

Since $a^{2}=c b^{2} c^{-1}$, the translation $a^{2}$ has both $\hat{k}$ and $c \cdot l$ as invariant geodesics (or perhaps $l$ and $c \cdot \hat{k}$ ). Two invariant geodesics of an isometry acting by a translation on both of them are at finite Hausdorff distance. Within a systolic complex this implies that either both $\hat{k}$ and $c \cdot l$ are quasi-convex or neither of them is (Els10, Proposition 3.12]). That contradicts the fact that $l$ is quasi-convex while $\hat{k}$ is not.

As we have just shown, the fundamental group of $K$ is not systolic; however, it is virtually systolic (there is a double covering $\bar{K}$, which is a $\mathcal{V H}$-complex, so $\pi_{1}(\bar{K})$ is systolic by Theorem 3.4). Now we use the complex $K$ to construct a square complex $S$ whose fundamental group is not even virtually systolic.

Let $E$ be the compact nonpositively curved $\mathcal{V H}$-complex which has no connected finite coverings, constructed by Wise in Wis07, Theorem 8.10]. Let $\sigma$ be any loop in $E$ consisting entirely of horizontal edges. We can subdivide the complex $K$ such that all loops $a, b$ and $c$ have the same combinatorial length as the loop $\sigma$. Now we define $\bar{E}$ and $\overline{\bar{E}}$ to be two copies of $E$ and let

$$
S=(E \cup \bar{E} \cup \overline{\bar{E}}) \cup K / \sim,
$$


where $\sim$ is the identification of $\sigma, \bar{\sigma}$ and $\overline{\bar{\sigma}}$ with $a, b$ and $c$, respectively. Then $S$ is a nonpositively curved square complex.

Theorem 4.2. The group $\pi_{1}(S)$ is not virtually systolic.

Proof. We first argue that $\pi_{1}(S)$ is not systolic. Since

$$
\pi_{1}(S)=\pi_{1}(K) *_{a=\sigma} \pi_{1}(E) *_{b=\bar{\sigma}} \pi_{1}(\bar{E}) *_{c=\overline{\bar{\sigma}}} \pi_{1}(\overline{\bar{E}})
$$

is an amalgam product, the inclusion $K \subset S$ induces an injection $\pi_{1}(K) \rightarrow \pi_{1}(S)$. To conclude that $\pi_{1}(S)$ is not systolic, we can recall the fact that a finitely presented subgroup of a torsion-free systolic group is systolic itself ([Wis05]), while $\pi_{1}(K)$ is not systolic (Theorem 4.1). An equivalent way of arriving at that conclusion is to repeat for $S$ the argument used for $K$ in the proof of Theorem 4.1.

To prove that $\pi_{1}(S)$ is not virtually systolic, we show that it has no finite-index subgroups (i.e. $S$ has no connected nontrivial finite coverings). Let $p: \tilde{S} \rightarrow S$ be a connected finite covering. Since $E \subset S$ has no connected nontrivial finite coverings, $p^{-1}(E)$ is a disjoint union of copies of $E$. In particular, any lift $\tilde{a}$ of the loop $a$ has the same length as $a$. The same holds for the loops $b$ and $c$. As $a, b$ and $c$, together with the three copies of $\pi_{1}(E)$, generate $\pi_{1}(S)$, this implies that $p$ is a trivial covering.

\section{ACKNOWLEDGEMENTS}

We thank Daniel Wise for motivating us and for his suggestions and discussions. We also thank the referee for a very thorough reading of the paper and many helpful remarks.

\section{REFERENCES}

[BM00] M. Burger and S. Mozes, Lattices in product of trees, Inst. Hautes Études Sci. Publ. Math. 92 (2000), 151-194 (2001). MR 1839489 (2002i:20042)

[Els09] T. Elsner, Flats and the flat torus theorem in systolic spaces, Geometry \& Topology 13 (2009), 661-698. MR2469526 (2009m:20065)

[Els10] _ Systolic groups with isolated flats (2010), submitted. Available online at http://www.math.uni.wroc.pl/〜elsner/papers/isolated-flats.pdf.

[JŚ06] T. Januszkiewicz and J. Świątkowski, Simplicial nonpositive curvature, Publ. Math. Inst. Hautes Études Sci. 104 (2006), 1-85. MR2264834 (2007j:53044)

[JŚ07] _ Filling invariants of systolic complexes and groups, Geometry \& Topology 11 (2007), 727-758. MR2302501(2008d:20079)

[Wis96] D. T. Wise, Non-positively curved squared complexes, aperiodic tilings, and non-residually finite groups (1996), Ph.D. thesis. MR2694733

[Wis03] A flat plane that is not the limit of periodic flat planes, Alg. Geom. Top. 3 (2003), 147-154. MR1997317 (2005a:20064)

[Wis05] _ Sixtolic complexes and their fundamental groups (2005), in preparation.

[Wis07] _ Complete square complexes, Comment. Math. Helv. 82(4) (2007), 683-724. $\operatorname{MR} 2341837(2009 \mathrm{c}: 20078)$

Mathematical Institute, University of WrocŁaw, Plac Grunwaldzki 2/4, 50-384 WroCŁAW, POLAND

E-mail address: elsner@math.uni.wroc.pl

Institute of Mathematics, Polish Academy of Sciences, Śniadeckich 8, 00-956 Warsaw, POLAND

E-mail address: pprzytyc@mimuw.edu.pl 